\title{
Mechanical Characteristic of a New-style Combined Parabolic Roof
}

\author{
Guo-an SUN ${ }^{1}$ Ai-liang SUN² \\ ${ }^{1}$ Lanzhou Jiaotong University, School of Civil Engineering Lanzhou 730070, China; \\ ${ }^{2}$ Lanzhou Jiaotong University, Lanzhou 730070,China
}

KEYWORD: combined thin shell; vibration; series solution

ABSTRACT: Folded plate structure is a dimensional one which is composed of a plurality of the strip plate. It is not only a bearing but also can support, material-saving and larger stiffness of thin-walled structure. It can be used as workshops, warehouses, stations, shops, schools, dwellings, pavilions, stadiums and the roves of other industrial and civil stories. The present buildings in the most of folded plate structure use folded plate roof of $\mathrm{V}$ shaped. The shape of it is so simple that affects the promotion of applied scope. This paper advances a hip-truncated combined parabolic cylinder roof structure that has good mechanical characteristic and beautiful appearance. The surface equation of a simply-supported hip-truncated combined parabolic cylinder roof is established by means of inclined coordinates and the step function. Then, the deflection and internal forces formulae of forced vibration of a simply-supported hip-truncated combined parabolic cylinder roof are derived from the theory of shallow shell vibration and variation method. The combined parabolic cylinder roof can also be solved by this method. Therefore two kinds of the questions of combined parabolic cylinder roof can be dealt using the method presented in the paper.

With the development of architectural technology, the format of architectural structure become newer and newer. The research of folded plate structure is attracted by many people. In recent years, there are so many researches about structural questions which belong to plate, cylindrical shell and spherical shell [1-3]. The researches of combined folded plate structure are paid close attention to people. RICKS[4] and CRISFIELD[5] present a method to solve the curving question of folded plate structure by iteration. LAI [6-8] presents some folded plate structures such as hip-truncated four-sloped folded plate roof, combined parabolic cylinder roof and folding $\mathrm{V}$ shaped folded plate roof and researches its internal forces, deflection and nonlinear characteristic by the nonlinear curving theory of shell.

Hip-truncated combined parabolic roof (Fig.1) and combined parabolic roof (Fig.2) can be seen in the real engineering, such as large-scale stadiums, auditoriums etc. But the dissertation of vibrated question in the aspect of shelled structure has not appeared yet. Based on Ref. [7], this paper presents combined parabolic roof and demonstrates the series solution of forced vibration.

\section{THE CURVED SURFACE EQUATION AND THE SHALLOW SHELL BENDING DIFFERENTIAL EQUATION}

The curved surface equation

The hip-truncated combined parabolic cylinder roof consists of plane A` B`C $C^{`} D^{`}$, curved

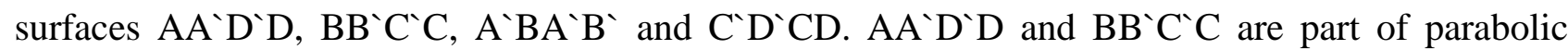
cylinder that parallels to the $\mathrm{x}$ axis, while $\mathrm{A}^{\prime} \mathrm{BA} \mathrm{A}^{\prime} \mathrm{B}^{`}$ and $\mathrm{C}^{\prime} \mathrm{D}^{`} \mathrm{CD}$ part of parabolic cylinder that parallels to the $y$ axis. In order to establish the surface equation of hip-truncated combined parabolic 
cylinder roof, the present study not only creates a global coordinate system, but also an oblique coordinate system (Fig.2), from which we can get the transformational relation between rectangular coordinate Oxy and oblique coordinate OXY.

$\left.\begin{array}{l}x=(Y+X) \cos \alpha \\ y=(Y-X) \sin \alpha\end{array}\right\}$

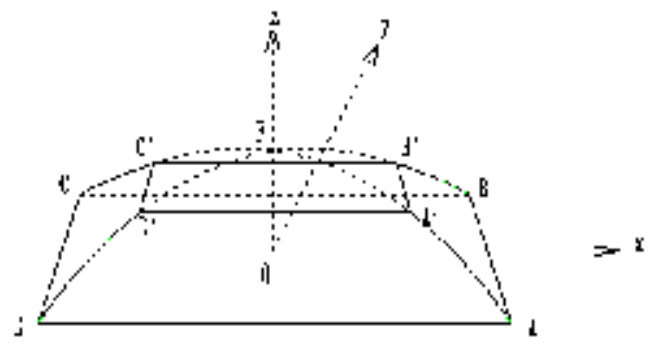

Fig.1 The hip-truncated combined parabolic cylinder roof

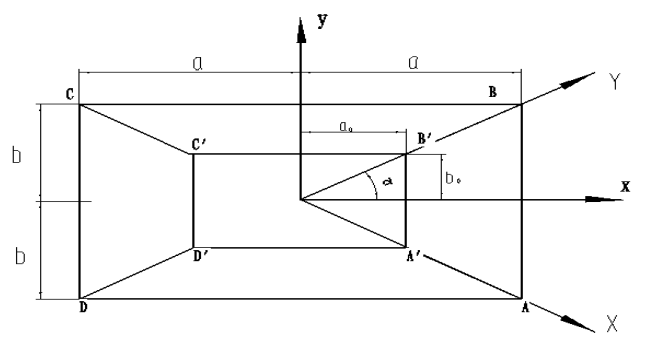

Fig.2 The vertical view of hip-truncated combined parabolic cylinder roof

Considering its symmetrical characteristics, a surface equation of the shallow shell in the first quadrant as follows:

$$
\begin{aligned}
Z= & f\left[1-u\left(y-b_{0}\right)\right] \cdot\left[1-u\left(x-a_{0}\right)\right]+u(X) u\left(x-a_{0}\right) f_{0}\left(1-\frac{x^{2}}{a^{2}}\right) \\
& +[1-u(X)] \cdot u\left(y-b_{0}\right) f_{0}\left(1-\frac{y^{2}}{b^{2}}\right)
\end{aligned}
$$

Where $\mathrm{f}$ is vector height, $f_{0}=\frac{f a^{2}}{a^{2}-a_{0}^{2}}=\frac{f b^{2}}{b^{2}-b_{0}^{2}}, u\left(x-a_{0}\right)$ is step function.

The shallow shell bending differential equation

With the help of derivative method for compound function and equation (1), we can get the degree of curve and torsion of the hip-truncated combined parabolic cylinder roof as follows:

$$
\begin{aligned}
k_{x}= & -\frac{\partial^{2} Z}{\partial x^{2}}=f\left[1-u\left(y-b_{0}\right)\right] \cdot \delta^{\prime}\left(x-a_{0}\right)-\delta^{\prime}(X) \frac{1}{4 \cos ^{2} \alpha} u\left(x-a_{0}\right) f_{0}\left(1-\frac{x^{2}}{a^{2}}\right) \\
& -\delta(X) \frac{1}{\cos \alpha} \delta\left(x-a_{0}\right) f_{0}\left(1-\frac{x^{2}}{a^{2}}\right)+\delta(X) \frac{1}{\cos \alpha} u\left(x-a_{0}\right) f_{0} \frac{2 x}{a^{2}} \\
& -u(X) \delta^{\prime}\left(x-a_{0}\right) f_{0}\left(1-\frac{x^{2}}{a^{2}}\right)+2 u(X) \delta\left(x-a_{0}\right) f_{0} \frac{2 x}{a^{2}} \\
& +u(X) u\left(x-a_{0}\right) \frac{2 f_{0}}{a^{2}}+\delta^{\prime}(X) \frac{1}{4 \cos ^{2} \alpha} u\left(y-b_{0}\right) f_{0}\left(1-\frac{y^{2}}{b^{2}}\right)
\end{aligned}
$$




$$
\begin{aligned}
k_{x y}= & -\frac{\partial^{2} Z}{\partial x \partial y}=-f \delta\left(y-b_{0}\right) \delta\left(x-a_{0}\right)+\delta^{\prime}(X) \frac{1}{2 \sin 2 \alpha} u\left(x-a_{0}\right) f_{0}\left(1-\frac{x^{2}}{a^{2}}\right) \\
& +\delta(X) \frac{1}{2 \sin \alpha} \delta\left(x-a_{0}\right) f_{0}\left(1-\frac{x^{2}}{a^{2}}\right)-\delta(X) u\left(x-a_{0}\right) \frac{f_{0}}{\sin \alpha} \frac{x}{a^{2}} \\
& -\delta^{\prime}(X) \frac{1}{2 \sin 2 \alpha} u\left(y-b_{0}\right) f_{0}\left(1-\frac{y^{2}}{b^{2}}\right)+\delta(X) \frac{1}{2 \cos \alpha} \delta\left(y-b_{0}\right) f_{0}\left(1-\frac{y^{2}}{b^{2}}\right) \\
& -\delta(X) \frac{1}{\cos \alpha} u\left(y-b_{0}\right) f_{0} \frac{y}{b^{2}} \\
k_{y} & =-\frac{\partial^{2} Z}{\partial y^{2}}=f^{\prime}\left(y-b_{0}\right)\left[1-u\left(x-a_{0}\right)\right] \\
- & \delta^{\prime}(X) \frac{1}{4 \sin { }^{2} \alpha} u\left(x-a_{0}\right) f_{0}\left(1-\frac{x^{2}}{a^{2}}\right) \\
& +\delta{ }^{\prime}(X) \frac{1}{4 \sin { }^{2} \alpha} u\left(y-b_{0}\right) f_{0}\left(1-\frac{y^{2}}{b^{2}}\right) \\
- & (X) \frac{1}{\sin \alpha} \delta\left(y-b_{0}\right) f_{0}\left(1-\frac{y^{2}}{b^{2}}\right) \\
& +\delta(X) \frac{1}{\sin \alpha} u\left(y-b_{0}\right) f_{0} \frac{2 y}{b^{2}} \\
- & {[1-u(X)] \delta^{\prime}\left(y-b_{0}\right) f_{0}\left(1-\frac{y^{2}}{b^{2}}\right) } \\
& +[1-u(X)] \delta\left(y-b_{0}\right) \frac{4 f_{0} y}{b^{2}} \\
+ & {[1+u(X)] u\left(y-b_{0}\right) \frac{2 f_{0}}{b^{2}} }
\end{aligned}
$$

When $\mathrm{f} / 2 \mathrm{~b}<=1 / 5$, shallow shell theory can be applied to this question, and the differential equation of variable camber shell is:

$$
\left.\begin{array}{c}
D \nabla^{4} W+\left(k_{x} \frac{\partial^{2}}{\partial y^{2}}+k_{y} \frac{\partial^{2}}{\partial x^{2}}-2 k_{x y} \frac{\partial^{2}}{\partial x \partial y}\right) \Phi+\rho h \frac{\partial^{2} W}{\partial t^{2}} \\
=-\left[q_{0}+\sum_{r=1}^{M} Q_{r} \delta\left(x-x_{r}, y-y_{r}\right)\right] \cos \omega t \\
\frac{1}{E h} \nabla^{4} \Phi-\left[\frac{\partial^{2}}{\partial y^{2}}\left(k_{x} W\right)+\frac{\partial^{2}}{\partial x^{2}}\left(k_{y} W\right)-2 \frac{\partial^{2}}{\partial x \partial y}\left(k_{x y} W\right)\right]=0
\end{array}\right\}
$$

$\mathrm{Q}_{0}, \mathrm{~h}$ and $\mathrm{E}$ in the equation refer to vertical uniformly distributed load, shell thickness and modulus of elasticity respectively. $D=E h^{3} / 12\left(1-\mu^{2}\right)$.

\section{THE SERIES SOLUTION OF VIBRATED RESPONSE}

For the four simply supported, we may suppose the progressive solution which meets the boundary condition as:

$$
\left.\begin{array}{c}
W=h \sum_{m=1}^{\infty} \sum_{n=1}^{\infty} A_{m n} \sin \frac{m \pi x}{2 a} \sin \frac{n \pi y}{2 b} \cos \omega t \\
\Phi=E h^{3} \sum_{m=1}^{\infty} \sum_{n=1}^{\infty} B_{m n} \sin \frac{m \pi x}{2 a} \sin \frac{n \pi y}{2 b} \cos \omega t
\end{array}\right\}
$$

Substitute the equation(3),(4),(5),(7) into equation (6) and change non-dual cosine series item 
into Dual cosine series, In series expansion, function $\delta$ and the following properties of its derivatives are applied[10].

$$
\begin{aligned}
& \int_{-\infty}^{\infty} \delta(x) f(x) d x=f(0), \quad \int_{-\infty}^{\infty} \delta^{\prime}(x) f(x) d x=-f^{\prime}(0) \\
& \delta[\Phi(x)]=\frac{\delta\left(x-x_{k}\right)}{\left|\Phi^{\prime}\left(x_{k}\right)\right|}
\end{aligned}
$$

Where $\mathrm{x}_{\mathrm{k}}$ is root of equation $\Phi(\mathrm{x})=0$. Then through coefficient comparison, we can get:

$$
\left.\begin{array}{c}
\sum_{m=1}^{k} \sum_{n=1}^{k} A_{m n}\left(K_{0}^{m n i j}+K_{1}^{m n i j}\right)+\sum_{m=1}^{k} \sum_{n=1}^{k} B_{m n} K_{2}^{m n i j}=K_{5}^{i j} \\
\sum_{m=1}^{k} \sum_{n=1}^{k} A_{m n} K_{3}^{m n i j}+\sum_{m=1}^{k} \sum_{n=1}^{k} B_{m n} K_{4}^{m n i j}=0
\end{array}\right\}
$$

Where

$$
\begin{aligned}
& K_{0}^{m n i j}=-\frac{\rho \omega^{2}}{E h^{2}} a b \delta_{m i} \delta_{n j} \\
& K_{1}^{m n i j}=\frac{a b}{12\left(1-\mu^{2}\right)}\left[\left(\frac{m \pi}{2 a}\right)^{4}+2\left(\frac{m \pi}{2 a}\right)^{2}\left(\frac{n \pi}{2 b}\right)^{2}+\left(\frac{n \pi}{2 b}\right)^{4}\right] \delta_{m i} \delta_{n j} \\
& K_{2}^{m i j}=-\frac{1}{h}\left(\frac{n \pi}{2 b}\right)^{2}\left\{\frac{f_{0}}{a b} \sum_{1=1}^{8} S_{1}-2 f Q_{2} Q_{3} \frac{b}{a}\right. \\
& +4 f\left[\frac{b}{(n+j) \pi} \sin \frac{(n+j) \pi b_{0}}{2 b}+Q_{1}\right] \cdot \frac{2 a_{0} Q_{3}}{a^{2}-a_{0}^{2}} \\
& +\mathrm{f}_{0}\left[\mathrm{H}_{9}+\frac{2}{(\mathrm{n}+\mathrm{j}) \pi \mathrm{a}}\left(\mathrm{H}_{2}-\mathrm{H}_{1}+\mathrm{H}_{6}-\mathrm{H}_{5}\right]\right\} \\
& -2 \frac{m n \pi^{2}}{4 a b h} \cdot\left[4 f \sin \frac{m \pi b_{0}}{2 b} \sin \frac{n \pi b_{0}}{2 b}\right. \\
& \left.\cos \frac{j \pi b_{0}}{2 b} \cos \frac{i \pi b_{0}}{2 b}-\frac{f_{0}}{b^{2}} \sum_{1=1}^{8}(-1)^{1} s_{1}\right] \\
& -\left(\frac{m \pi}{2 a}\right)^{2} \frac{1}{h}\left\{2 f \left[\frac{2 a}{(m+i) \pi} \sin \frac{(m+i) \pi a_{0}}{2 a}\right.\right. \\
& \left.+2 T_{1}\right] \cdot \frac{b_{0}}{b^{2}-b_{0}^{2}} Q_{2}+\frac{a f_{0}}{b^{3}} \sum_{1=1}^{8} S_{1} \\
& \left.-2 \frac{a f}{b} Q_{2} Q_{3}+f_{0}\left[-\frac{2 a}{(m+i) \pi b^{2}} \cdot \sum_{l=1}^{4} H_{1}+V_{9}\right]\right\} \\
& K_{3}^{m n i j}=-K_{2}^{m n i j} \\
& K_{4}^{m n i j}=12\left(1-\mu^{2}\right) \cdot K_{1}^{m n i j} \\
& c_{l}=d_{l} \times b \quad(l=1,2, \ldots 8) \\
& e_{l}=d_{l} \cdot b_{0} \quad(l=1,2, \cdots, 8)
\end{aligned}
$$




$$
\begin{aligned}
& d_{1}=\frac{\pi}{2 b}(m+i+n+j), d_{2}=\frac{\pi}{2 b}(m+i-n-j) \\
& d_{3}=\frac{\pi}{2 b}(m+i+n-j), d_{4}=\frac{\pi}{2 b}(m+i-n+j) \\
& d_{5}=\frac{\pi}{2 b}(m-i+n+j), d_{6}=\frac{\pi}{2 b}(m-i-n-j) \\
& d_{7}=\frac{\pi}{2 b}(m-i+n-j), d_{8}=\frac{\pi}{2 b}(m-i-n+j)
\end{aligned}
$$$$
H_{l}=\left\{\begin{array}{lc}
\frac{1}{d_{l}}\left(\cos c_{l}-\cos e_{l}\right) & d_{l} \neq 0 \\
0 & d_{l}=0
\end{array} \quad l=(1,2, \cdots, 8)\right.
$$$$
H_{9}=\left\{\begin{array}{cc}
\frac{2}{(n-j) \pi a}\left(H_{4}-H_{3}+H_{8}-H_{7}\right) \quad n \neq j \\
\left\{H_{10}+\frac{8 b}{a(m-j)^{2} \pi^{2}}\left[\cos \frac{(m-j) \pi}{2}-\cos \frac{(m-i) \pi a_{0}}{2 a}\right]\right. \\
\left.-\frac{4 b a_{0}}{(m-i) \pi a^{2}} \sin \frac{(m-i) \pi a_{0}}{2 a}\right\} & n=j, m \neq i \\
H_{10}+\frac{b}{a^{3}}\left(a^{2}-a_{0}^{2}\right) & n=j, m=i
\end{array}\right.
$$$$
\mathrm{H}_{10}=\frac{2 b}{\mathrm{a}}\left\{\frac{4}{(\mathrm{~m}+\mathrm{i})^{2} \pi^{2}}\left[\cos \frac{(\mathrm{m}+\mathrm{i}) \pi}{2}-\cos \frac{(\mathrm{m}+\mathrm{i}) \pi \mathrm{a}_{0}}{2 \mathrm{a}}\right]\right.
$$$$
\left.\frac{2 a_{0}}{(m+i) \pi a} \sin \frac{(m+i) \pi a_{0}}{2 a}\right\}
$$$$
Q_{1}= \begin{cases}\frac{b}{(n-j) \pi} \sin \frac{(n-j) \pi b_{0}}{2 b} & n \neq j \\ \frac{b_{0}}{2} & n=j\end{cases}
$$$$
Q_{2}=2 \cos \frac{n \pi a_{0}}{2 a} \cos \frac{j \pi a_{0}}{2 a}, Q_{3}=\cos \frac{m \pi a_{0}}{2 a} \cos \frac{i \pi a_{0}}{2 a}
$$$$
S_{l}=\left\{\begin{array}{lcc}
\frac{1}{d_{l}^{2}}\left(\cos c_{l}-\cos e_{l}\right)+\frac{1}{d_{l}}\left(b \sin c_{l}-b_{0} \sin e_{l}\right) & d_{l} \neq 0 \\
\frac{b^{2}-b_{0}^{2}}{2} & d_{l}=0 & l=(1,2, \cdots, 8)
\end{array}\right.
$$

$T_{1}= \begin{cases}\frac{a}{(m-i) \pi} \sin \frac{(m-i) \pi a_{0}}{2 a} & m \neq i \\ \frac{a_{0}}{2} & m=i\end{cases}$ 


$$
\begin{aligned}
& V_{9}=\left\{\begin{array}{cc}
-\frac{2 a}{(m-i) \pi b^{2}} \sum_{l=5}^{8} H_{l} & m \neq i \\
\left\{V_{10}+\frac{8 a}{b(n-i)^{2} \pi^{2}}\left[\cos \frac{(n-j) \pi}{2}-\cos \frac{(n-j) \pi b_{0}}{2 b}\right]\right. \\
\left.-\frac{4 a b_{0}}{(n-j) \pi b^{2}} \sin \frac{(n-j) \pi b_{0}}{2 b}\right\} & m=i, n \neq j \\
V_{10}+\frac{a}{b^{3}}\left(b^{2}-b_{0}^{2}\right) & m=i, n=j
\end{array}\right. \\
& V_{10}=\frac{2 a}{b}\left\{\frac{4}{(n+j)^{2} \pi^{2}}\left[\cos \frac{(n+j) \pi}{2}-\cos \frac{(n+j) \pi b_{0}}{2 b}\right]\right. \\
& \left.-\frac{2 b_{0}}{(n+j) \pi b} \sin \frac{(n+j) \pi b_{0}}{2 b}\right\} \\
& K_{5}^{i j}=-\frac{4 a b q_{0}}{\operatorname{Eh}^{4} i j \pi^{2}}(1-\cos i \pi)(1-\cos j \pi) \\
& -\frac{1}{E^{4}} \sum_{r=1}^{M} Q_{r} \sin \frac{i \pi x_{r}}{2 a} \sin \frac{j \pi y_{r}}{2 b}
\end{aligned}
$$

Respectively, take $\mathrm{i}, \mathrm{j}=1,2,3, \ldots \ldots \mathrm{k}$, with equation (5) forms a $2 \times \mathrm{K} \times \mathrm{K}$ order linear equation system. Solve it, we get the value of Amn and Bmn, substitute into equation (4), displacement and stress function, and then in this way, The Internal Forces and deflection of a hip-truncated combined parabolic cylinder roof an be figured out.

When the interfering force changes in the way of the non simple harmonic periodic function, we can expand the interfering force into Fourier series. Then calculates each response and overlies them in the way that this paper advances. When we calculate the frequency of free vibration, take $\mathrm{q}_{0}=0, \quad \mathrm{Qr}=0(\mathrm{r}=1,2, \ldots, \mathrm{M})$ into equation (5), let coefficient determinant equal 0 and acquire frequency equation of a hip-truncated combined parabolic roof. Finally we can solve the frequency of free vibration. When we calculate the Internal forces and deflection in the condition of symmetrical load Qr acting on random position, take $\omega=0$ into equation (5). When $\mathrm{a}_{0}=\mathrm{b}_{\mathrm{o}}=0$, the hip-truncated combined parabolic cylinder roof which is demonstrated in Fig.1 becomes combined parabolic cylinder roof. It can achieve the frequency equations of combined parabolic cylinder roof in the condition of free vibration and the solution of statics question in the condition of symmetrical load and concentrated force. Up to six aspects of problems that include forced vibration, free vibration and static question etc. can be dealt using the method presented in the paper.

\section{DEMONSTRATIVE EXAMPLE}

Suppose there is a four-edged simply-supported hip-truncated combined parabolic roof, $a=b=3.5 m, f_{0}=1.3 m, a_{0}=b_{0}=0.94 m, h_{0}=0.05 m$, load $q(t)=2.5 \cos 200 t \mathrm{KN} / \mathrm{m}^{2}$, the modulus of elasticity $E=3 \times 10^{7} \mathrm{KN} / \mathrm{m}^{2}$, the density $\rho=2.5 \times 10^{3} \mathrm{~kg} / \mathrm{m}^{2}$. Apply the method in this article, we can get the displacement as shown in the section of Fig. 3 and surface stresses $\sigma_{x} 、 \sigma_{y}$ as 
shown in Fig .4 6(full line). Suppose $\omega=0, q(t)=2.5 \mathrm{KN} / \mathrm{m}^{2}$ apply the method in this article, we can calculate the displacement as shown in the section of Fig. 3 and surface stresses $\sigma_{x} 、 \sigma_{y}$ as shown in Fig .4 6(dotted line) in the condition of static acting force.

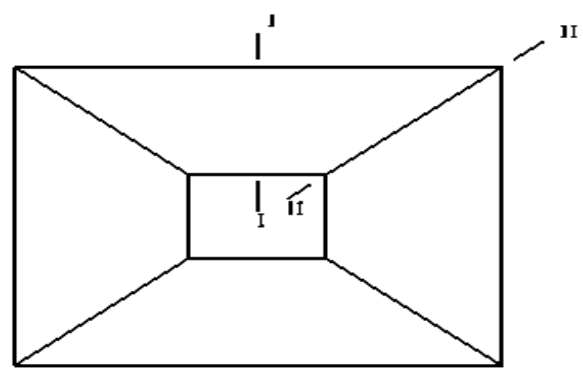

Fig.3 The sectional position of roof
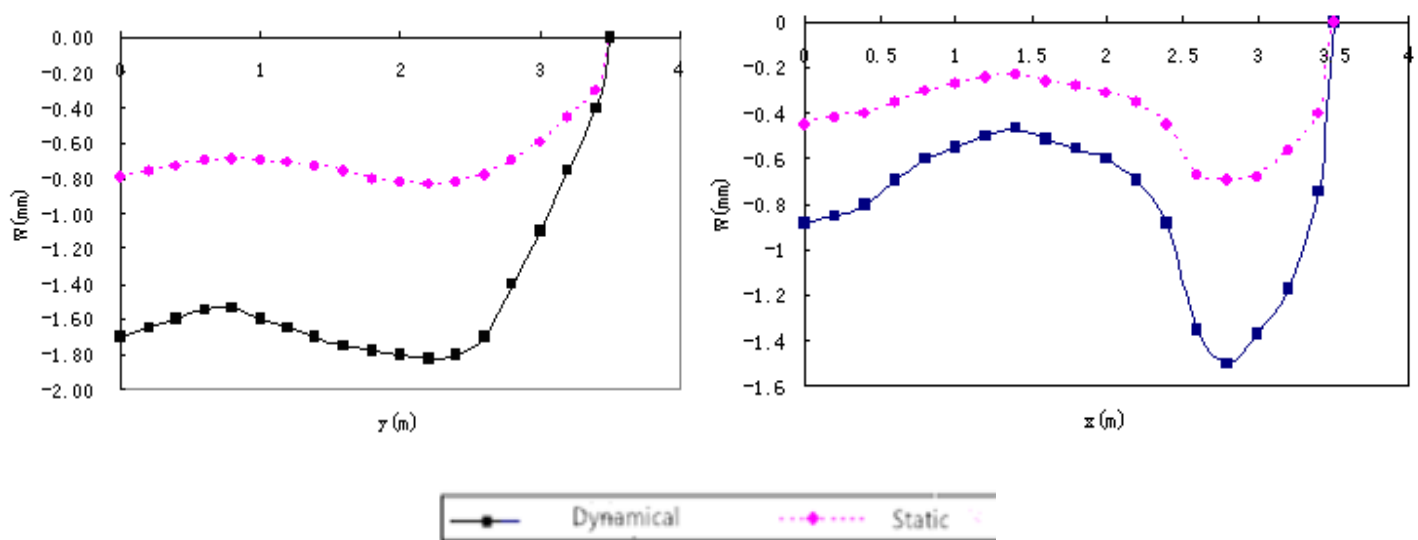

Fig.4 I-I sectional and II-II sectional displacement
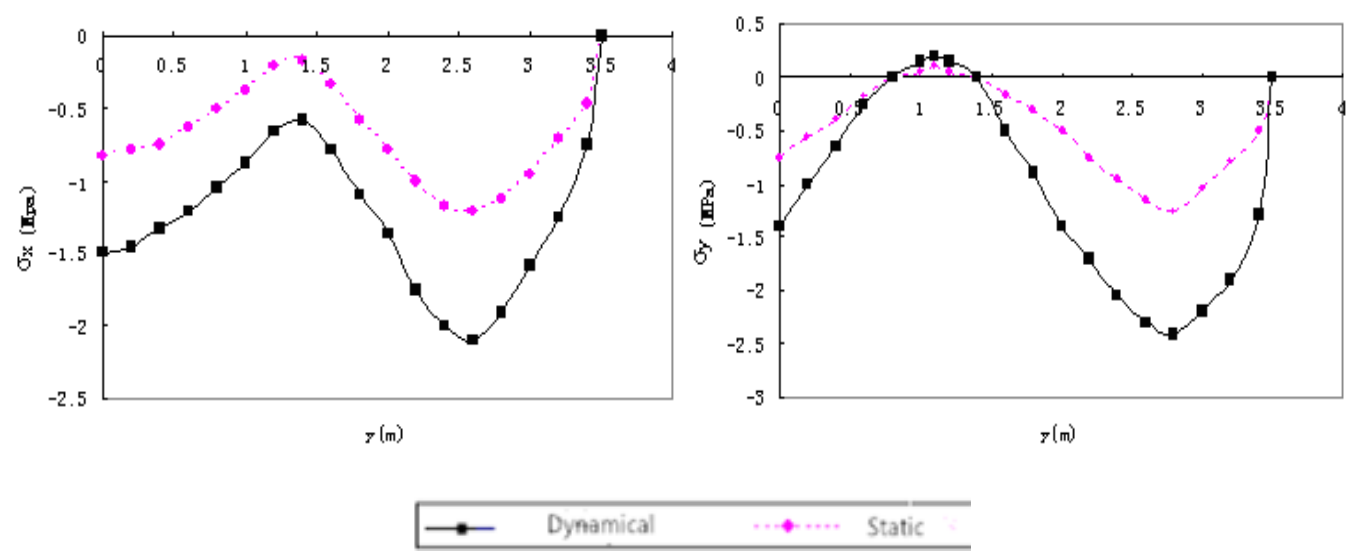

Fig.5 I-I sectional stress $\sigma_{\mathrm{x}}$ and $\sigma_{\mathrm{y}}$ 


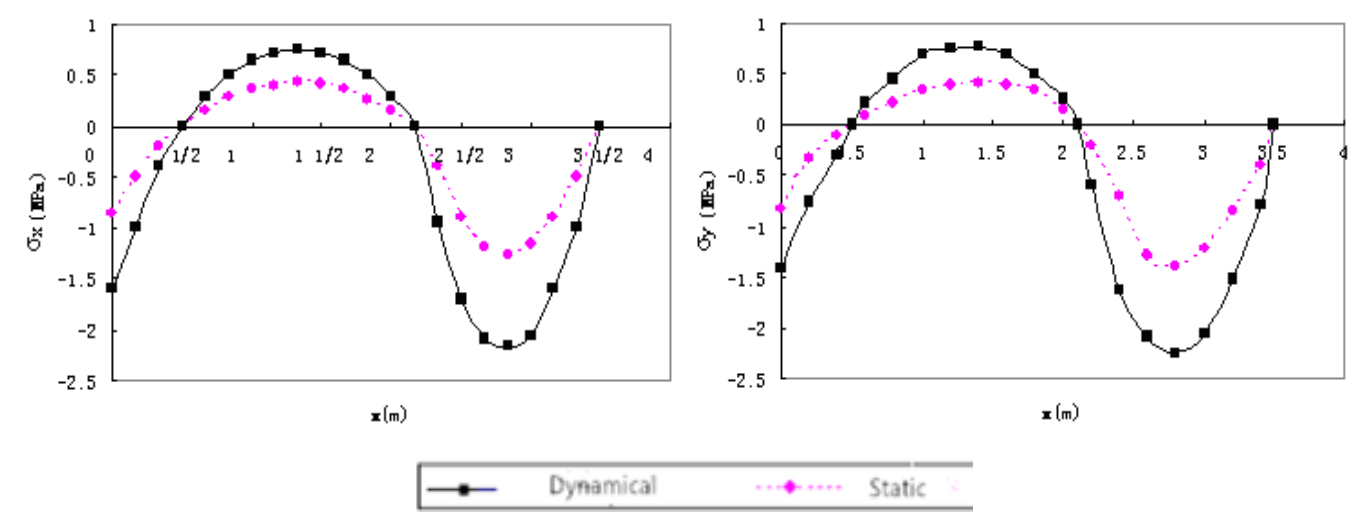

Fig.6 II-II sectional stress $\sigma_{\mathrm{x}}$ and $\sigma_{\mathrm{y}}$

According to calculation, we can find that the displacement in the condition of vibration is much larger than the one in the condition of static force. The maximal displacement in the condition of vibration appears in the position of I-I sectional. The coordinate is $(0,2.4 \mathrm{~m})$ and the numerical value is $-1.824 \mathrm{~mm}$; The maximal stress appears in the position of I-I sectional. The coordinate is

$(0,2.8 \mathrm{~m})$ and the numerical value is $\sigma_{y}=-2.43 \mathrm{MPa}$. It is obvious that the maximal displacement and the maximal stress appears in the position of I-I sectional. Therefore we can strengthen the stability in the position of I-I sectional.

\section{CONCLUSION}

This paper presents a good mechanical performance, the appearance of beautiful a hip-truncated combined parabolic cylinder roof structure, The surface equation of a simply-supported hip-truncated combined parabolic cylinder roof is established by means of inclined coordinates and the step function. Then, the deflection and internal forces formulae of forced vibration of a simply-supported hip-truncated combined parabolic cylinder roof are derived from the theory of shallow shell vibration and variation method. The combined parabolic cylinder roof can also be solved by this method. It can be applied in the initial design and many-sided comparative.

\section{REFERENCE}

[1] Jun CHOU, Shu-yong WANG, Jun-yong GAO et al. Research on forced vibrations of simply supported rectangular plate with absorber[J]. Journal of Guangxi University ( Nat Sci Ed), 2005 , $30(3): 229 \sim 232$

[2] Lin LI. An exact solution for free vibration of toroidal thin shell[J]. Journal of Aerospace Power, $1997,12(2): 117 \sim 121$

[3] Wei-dong ZHAO, Ping QIU, Xiao WU et al. Geometrically nonlinear free vibration of a shallow spherical shell under uniform pressure[J]. Journal of Lanzhou University of Technology, 2010 , 36 ( 4 ) : 168 171

[4] RIKS E. An incremental approach to the solution of snapping and buckling problems [J]. International Journal of Solids Structures, 1979, 15(7):529-551 
[5] CRISFIELD MA.A fast incremental/iterative solution procedure that handles snap-through [J].Computers and Structures,1981,13(1):55-62]

[6] Yuan-ming LAI. Deflection and internal forced of a simply supported truncated hip roof [J]. Chinese journal Civil Engineering, 1995, 28 ( 1 ) : 33 39

[7] Yuan-ming LAI ,Ya-ping WU. Linear \& nonlinear analysis of a combined parabolical cylinder roof[J]. Computers \&Structures, $1994,53(5): 1067-1072$

[8] Yuan-ming LAI. Analytical solution for forced vibration of a simply-supported V-shaped folded plate roof [J]. Thin-Walled Structures, 2002, 40(3):215-224

[9] Xiang-sheng CHENG. Theory of applied plate[M].Jinan: Sandong Science and Technology Press, 1989

[10]Kun-miao LIANG. Method of mathematics and physics[M]. Beijing :People Educational Press, 1978 